Journal of Clinical and

Translational Science

www.cambridge.org/cts

\section{Education \\ Research Article}

Cite this article: Enders FT, Golembiewski EH, Orellana M, Silvano CJ, Sloan J, and Balls-Berry J. The hidden curriculum in health care academia: An exploratory study for the development of an action plan for the inclusion of diverse trainees. Journal of Clinical and Translational Science 5: e203, 1-7. doi: 10.1017/ cts.2021.867

Received: 13 August 2021

Revised: 4 October 2021

Accepted: 4 October 2021

\section{Keywords:}

Hidden curriculum; mentorship; doctoral education; diversity; equity; inclusion; professional development

\section{Address for correspondence:}

F. T. Enders, PhD, MPH, Division of Biomedical Statistics and Informatics, Department of Quantitative Health Sciences, Mayo Clinic, Rochester, MN, USA.

Email: enders.felicity@mayo.edu

\title{
The hidden curriculum in health care academia: An exploratory study for the development of an action plan for the inclusion of diverse trainees
}

\author{
Felicity T. Enders ${ }^{1}$ (D) Elizabeth H. Golembiewski ${ }^{2}$ (D) , Minerva Orellana $^{3}(\mathbb{D}$, \\ Carmen J. Silvano ${ }^{3}$, Jeff Sloan ${ }^{1}$ and Joyce Balls-Berry ${ }^{4}$
}

${ }^{1}$ Department of Quantitative Health Sciences, Mayo Clinic, Rochester, MN, USA; ${ }^{2}$ Knowledge and Evaluation Research (KER) Unit, Mayo Clinic, Rochester, MN, USA; ${ }^{3} \mathrm{Clinical}$ and Translational Science Track, Mayo Clinic Graduate School of Biomedical Sciences, Mayo Clinic, Rochester, MN, USA and ${ }^{4}$ Department of Neurology, Washington University, St. Louis, MO, USA

\begin{abstract}
Introduction: The hidden curriculum encompasses the norms, values, and behaviors within a learning environment. Navigating the hidden curricula of academia is crucial for doctoral trainees, particularly those from underrepresented backgrounds. Faculty mentors have an important role in helping trainees uncover and cope with the hidden curriculum. The purpose of this paper is to explore perceptions of the hidden curriculum among diverse doctoral trainees and mentors. Methods: Following a presentation on the hidden curriculum at the Association for Clinical and Translational Science annual meeting in March 2021, attendees were asked to brainstorm ideas for diverse trainees and their mentors. Breakout room discussions were held for specific hidden curriculum topics; participants voted on which topics to discuss from a list of topics defined during the presentation. Ideas from these discussions were presented to the larger group to upvote. Results: Participants $(n=116)$ voted to discuss the following hidden curriculum topics: "coping with bias," "assertive communication," "knowing how things work," and "developing a career." Many suggestions emphasized the role of institutions in empowering mentors to help diverse trainees and, more generally, to meaningfully support policies and programs that facilitate the career success of trainees and faculty from underrepresented backgrounds. Conclusions: This work generated a list of suggested action items for trainees, mentors, and institutions to ameliorate the hidden curricula of academia, especially for diverse trainees. However, institutions need to support changes that will facilitate these discussions as well as more broadly enable the success of faculty and students from diverse backgrounds.
\end{abstract}

(c) The Author(s), 2021. Published by Cambridge University Press on behalf of The Association for Clinical and Translational Science. This is an Open Access article, distributed under the terms of the Creative Commons AttributionNonCommercial-NoDerivatives licence (https:// creativecommons.org/licenses/by-nc-nd/4.0/), which permits non-commercial re-use, distribution, and reproduction in any medium, provided the original work is unaltered and is properly cited. The written permission of Cambridge University Press must be obtained for commercial re-use or in order to create a derivative work.



Analysis. Adrocacy. Action.
"Education is about inviting every single person who enters a school to realize his or her relatively boundless potential in all areas of worthwhile human endeavor. It is concerned with more than grades, attendance, and academic achievement. It is concerned with the process of becoming a decent and productive human being."

William Watson Purkey

\section{Introduction}

The term "hidden curriculum" refers to the unspoken norms, values, and behaviors that exist within a learning environment $[1,2]$. In contrast to formal, explicit rules and educational expectations of the classroom, laboratory, or training program, these hidden curricula are rarely planned or articulated, leaving trainees to discern their existence and relative importance on their own or with the help of mentors [2]. Specifically, in the context of health sciences doctoral training programs, a variety of hidden curricula may be manifest within an institution, department, or academic discipline that weigh heavily on a student's ability to not only succeed but thrive as an early career researcher [1] and beyond [3]. In doctoral training settings, these hidden curricula encompass a range of "ways of being," from conducting and disseminating research (e.g., how do I navigate authorship order on a manuscript?) to personal bearing and behaviors (e.g., how do I ask a question during a scholarly presentation without seeming confrontational? How and to whom do I tell my needs and frustrations?).

It is given that the ability to deal with hidden curricula is highly variable. Beyond natural individual variation, certain groups of doctoral trainees may lack the mentorship and guidance necessary to unpack the hidden curricula within their institution or field. In particular, trainees who come from "outside" the majority system are often at a disadvantage in understanding and dealing with hidden curricula. Classism, racism, sexism, heteronormativism, and ableism establish, exacerbate, and perpetuate hidden systems of power [4-7]. Institutions ideally should challenge and reform the exclusionary cultures that exist within their walls, but meaningful change 
has historically often been slow to manifest itself. To succeed in noninclusive environments, individuals from diverse backgrounds may need additional help to understand how to navigate the unwritten rules and norms of an academic environment. For example, students from nonmajority backgrounds may need more help than majority trainees in understanding the subtle ways in which how a communication or presentation may be perceived by the recipient (e.g., when to contact mentors and collaborators, what tone to utilize in email and other communications, how to modify the tone to fit the recipient's role) $[2,8]$. In addition, faculty mentors may provide more support for trainees with whom they feel greater affinity [8], leaving students from underrepresented backgrounds at a disadvantage in navigating the hidden curriculum of academia.

The hidden curriculum has been well studied in medical school education, with hundreds of papers on the topic published since 1980 [9], but is novel in the context of academic health sciences research. Medical training has evolved to include interpersonal skills, listening to the patient, empathy, and other hidden curricula to produce a more capable and rounded medical professional. However, health sciences research programs typically do not teach basic communication or other interpersonal skills needed for a successful career in academia. A common complaint of leaders of health care institutions, especially pharmaceutical companies, is that they have to provide supplementary training for new graduates before they can engage in productive research [10-12]. In addition, improvements specifically to communicating diversityrelated hidden curricula - for example, teaching junior faculty how to cope with racism in the workplace - are also aligned with improving the overall culture within institutions, which is thought to improve both recruitment and retention relating to diversity [13]. The objective of this paper is to present novel insights into perceptions of the hidden curriculum among those whom it affects the most - doctoral trainees and their mentors, with an emphasis on trainees from diverse backgrounds. Specifically, we describe the findings of an interactive presentation and workshop on diversityrelated hidden curricula. Participants were asked to reflect on and discuss the role of the hidden curriculum in doctoral training and to brainstorm solutions for diverse trainees and their mentors to unpack and overcome diversity-related hidden curricula. We believe these findings will be of interest to doctoral program administrators, faculty mentors, and trainees as a resource to improve retention and success of doctoral trainees from diverse backgrounds and guide future interventions in this area.

\section{Materials and Methods}

\section{Hidden Curriculum Content Development}

We first constructed a series of items on learner perceptions of hidden curriculum topics to inform the content of the larger presentation and workshop. A group of 22 trainees currently enrolled in the Mayo Clinic TL1 Clinical and Translational Science (CTS) predoctoral training program were asked to rate the relative importance of 10 hidden curriculum topics for a hypothetical majority trainee and the relative added importance for a diverse trainee over and above what is needed for a majority trainee. Respondents were asked to rate how important each hidden curriculum topic (from list in Table 1) was for six distinct groups: majority trainees and diverse trainees; new mentors of majority trainees and new mentors of diverse trainees; and CTS mentors of majority trainees and
Table 1. Select research and diversity-related hidden curriculum topics and their descriptions

\begin{tabular}{|c|c|}
\hline $\begin{array}{l}\text { Hidden curriculum } \\
\text { topic }\end{array}$ & Description \\
\hline Research logistics & $\begin{array}{l}\text { How was a project done and who helped it } \\
\text { happen }\end{array}$ \\
\hline $\begin{array}{l}\text { Research } \\
\text { communication* }\end{array}$ & $\begin{array}{l}\text { Written and oral communication needed for } \\
\text { research }\end{array}$ \\
\hline Research strategy & How to plan a long-term research strategy \\
\hline Withstand setbacks & How to plan for and cope with setbacks \\
\hline $\begin{array}{l}\text { Knowing how things } \\
\text { work }^{*}\end{array}$ & $\begin{array}{l}\text { Institutional norms and the nuts and bolts of } \\
\text { research }\end{array}$ \\
\hline Getting things done & $\begin{array}{l}\text { Use time management to complete the critical } \\
\text { and the mundane }\end{array}$ \\
\hline $\begin{array}{l}\text { Networking and } \\
\text { external visibility }\end{array}$ & How to build a career intentionally \\
\hline Work-life balance & $\begin{array}{l}\text { Skills and negotiation at home to achieve } \\
\text { satisfaction in both }\end{array}$ \\
\hline $\begin{array}{l}\text { Assertive } \\
\text { communication* }\end{array}$ & How to be assertive, not aggressive \\
\hline Confidence $^{\star}$ & $\begin{array}{l}\text { Understand the impact on confident thoughts } \\
\text { and behaviors of a lifetime facing bias }\end{array}$ \\
\hline $\begin{array}{l}\text { Respond to specific } \\
\text { biases }\end{array}$ & $\begin{array}{l}\text { Understand challenges trainees may face \& } \\
\text { attempt to mitigate them }\end{array}$ \\
\hline Cope with bias* & $\begin{array}{l}\text { Observe and act on potential bias in others, } \\
\text { both overt and joking }\end{array}$ \\
\hline Focus on research & $\begin{array}{l}\text { Understand the diversity tax; plan when to say } \\
\text { yes and when to decline }\end{array}$ \\
\hline Develop a career ${ }^{\star}$ & $\begin{array}{l}\text { Build toward a career intentionally, including } \\
\text { knowing opportunities, what they require, and } \\
\text { networking }\end{array}$ \\
\hline
\end{tabular}

Starred topics are those presented in the ACTS conference presentation and workshop.

CTS mentors of diverse trainees. Respondents rated each hidden curriculum topic on a scale from 0 to 3 to indicate its relative importance for a given group. For the questions about diverse trainees and mentors of diverse trainees, respondents were asked to think about any excess need associated with hidden curricula for these groups.

The 10 hidden curriculum topics were developed based upon available evidence on pervasive diversity-related norms and cultural expectations in professional settings [14-17]. In particular, Dr Byars-Winston developed a theoretical model for barriers to promotion and leadership for diverse individuals and women which formed a basis for the curriculum topics used in our study [18]. Specifically, practical approaches to coping with hidden curriculum [15-17] focus on increasing self-efficacy, which ByarsWinston et al. found to be the primary driver of scientific identity, outcome expectations, and career. Each topic was introduced with research-based concepts upon which training could be based. For instance, written and oral research communication included not only peer-reviewed papers but also emails, for which a trainee needed to identify recipients to include, when to contact them, and what tone to utilize. The expectation is that, with faculty guidance, trainees can edit standard examples with faculty feedback and then practice reading their own writing aloud to identify problems. 


\section{Conference Presentation and Workshop}

In March 2021, the first author (FTE) presented an interactive session on diversity-related research hidden curriculum at the Association for Clinical and Translational Science annual meeting. The focus of the presentation content was on the role of the hidden curriculum for trainees and their faculty mentors, its particular importance for trainees from diverse backgrounds, and a summary of findings from the preliminary data described above. Grounded in results from the content construction process with Mayo Clinic trainees, the conference presentation focused on several specific hidden curriculum topics, including research communication, or the written and oral communication norms within research; knowing how things work, broadly defined as institutional norms and the nuts and bolts of research; developing a career, or building toward a career intentionally, including knowing opportunities, what they require, and continued networking; confidence, including developing confident thoughts and behaviors to overcome a lifetime of prior environments; assertive communication, or how to be assertive without being seen as aggressive; and coping with bias, or how to identify and act on potential bias in others.

Throughout the session, participants were asked to respond to several questions using Poll Everywhere, a real-time polling software for presentations. All poll responses were voluntary and anonymous. Participants were first asked to report whether they had previous familiarity with the term "hidden curriculum" ("Yes, I am familiar with the idea of hidden curriculum"; "Yes, but I only learned about hidden curriculum from information about this session"; or "No, hidden curriculum is a brand new concept to me") and to respond to the prompt "What does hidden curriculum look like to you?" For this question, free-text responses were accepted, which other session participants could then upvote or downvote using the polling software.

From the list in Table 1, participants were asked to vote on the top 1-2 specific hidden curriculum topics that they would like to discuss in small groups. For each topic, there were separate breakout rooms to brainstorm ideas and strategies for (1) trainees or (2) mentors of trainees. Both trainees and faculty were encouraged to take part in each group. An additional "wildcard" breakout group was also created for participants wishing to discuss hidden curriculum topics not addressed in other rooms. Each participant could self-select into their preferred discussion group, regardless of the original topic for which they voted. All breakout rooms were moderated by Mayo Clinic pre- and postdoctoral trainees. For each group, participants were given 20 min to discuss while the moderator took notes and recorded the top 3-5 ideas from each brainstorming session. Once the larger group had reconvened, moderators from each of the groups were asked to record their group's top ideas in a poll to allow members of the larger group to upvote or downvote different ideas. Results of these polls produced a list of potential actions and ideas with their relative importance based on number of upvotes.

\section{Results}

\section{Hidden Curriculum Content Development}

Fig. 1 shows results from polling of 22 trainees currently enrolled in the Mayo Clinic TL1 CTS predoctoral training program, which demonstrate significant need for training in targeted hidden curriculum for all trainees, and additional need for diverse trainees. Moderate need is also identified in targeted hidden curriculum training for CTS mentors relevant to mentoring generally, with
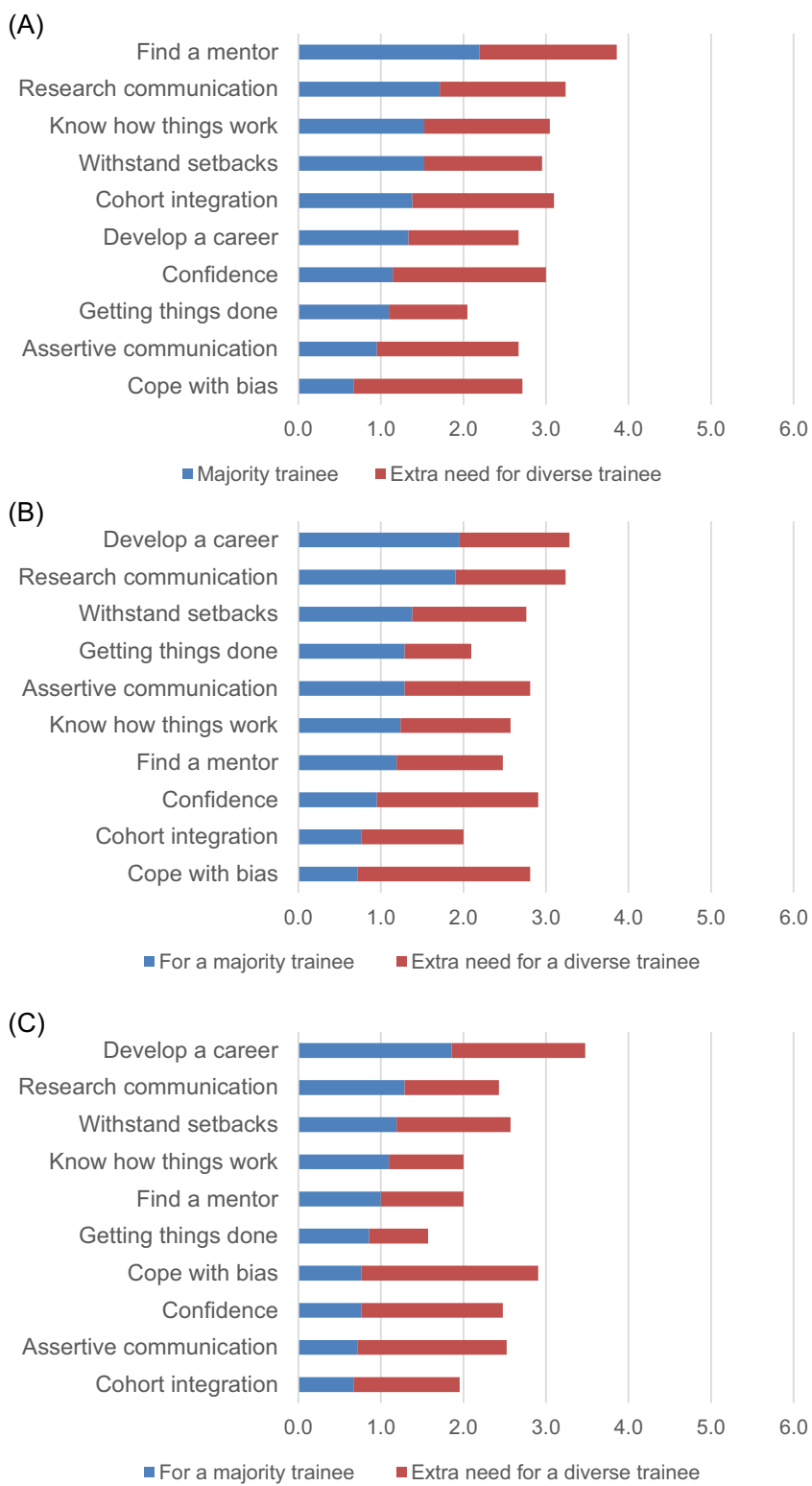

Fig. 1. Preliminary data on doctoral student perceptions of trainee and mentor hidden curriculum needs $(n=22)$. (A) What does a trainee need to know at the start of their program? (B) What does a new mentor with no prior knowledge need to know? (C) What does a typical CTS mentor need to learn? Note: Relative "need" for majority trainees and extra "need" for diverse trainees were on a 0-3 scale, respectively, for a total of 6 points possible for each topic ( 3 for the need for a majority trainee, 3 for the additional need for a diverse trainee). When the respondent perceived that the need was identical for a majority and diverse trainee, they were instructed that the need for a diverse trainee should be 0 .

significant additional need for hidden curriculum training that focuses on mentoring diverse trainees. Specifically, hidden curriculum topics for which respondents identified extra need for diverse trainees and their mentors included developing an assertive communication style, confidence, and coping with bias.

\section{Conference Presentation and Workshop}

A total of 116 attendees participated in at least one polling activity. Nearly three-quarters of participants identified as female $(n=58$; $73 \%$ ), while over one-third reported that their cultural heritage is outside the United States $(n=27 ; 34 \%)$; see Table 2 for overview of 
Table 2. Hidden curriculum workshop participant diversity characteristics ( $n=79)$

\begin{tabular}{ll}
\hline Diversity group & $\mathrm{N}(\%)$ \\
\hline My gender identity is female & $58(73.4)$ \\
\hline My cultural heritage is outside the United States & $27(34.2)$ \\
\hline In my family, I was in the first generation to attend college & $23(29.1)$ \\
\hline My background includes financial disparity & $23(29.1)$ \\
\hline I am an underrepresented minority: Black, Hispanic, Native & $18(22.8)$ \\
American, Alaskan Native, Native Hawaiian, Pacific Islander & \\
\hline I am another racial minority not listed above & $14(17.7)$ \\
\hline English was not my first language & $13(16.5)$ \\
\hline I am a sexual or gender minority & $11(13.9)$ \\
\hline None of the above apply to me & $5(6.3)$ \\
\hline
\end{tabular}

participant diversity characteristics. The highest proportion of respondents identified as faculty (34\%), TL1 program staff (20\%), or graduate students (19\%). Participants were split on prior knowledge of the hidden curriculum - $44 \%$ reported that they were previously familiar with the idea, while an equal amount of $44 \%$ stated that the hidden curriculum was a brand new concept. When respondents were asked to enter free text in response to the question "What does hidden curriculum look like to you?" the most upvoted answers were "unwritten rule" (44 upvotes), "behaviors/norms that aren't explicitly taught" (41 upvotes), "dominant culture's norms, values, beliefs embedded" (39 upvotes), and "knowing the system" (37 upvotes).

When respondents were asked to identify the 1-2 specific hidden curriculum topics they wished to brainstorm about in breakout groups, the top selections were "coping with bias" ( $\mathrm{n}=32 ; 27 \%)$, "assertive communication" ( $\mathrm{n}=28 ; 24 \%)$, "knowing how things work" $(\mathrm{n}=24 ; 21 \%)$, and "developing a career" $(\mathrm{n}=15 ; 13 \%)$; see Table 1 for descriptions of each hidden curriculum topic. In addition, some respondents participated in a "wildcard" group tasked with brainstorming ideas for trainees and mentors in the context of hidden curriculum explicitly outside the topic areas selected for other breakout groups. Fig. 2 presents the top ideas generated by each breakout group and the number of upvotes for each idea.

\section{Breakout Group Session Results}

\section{Coping with bias}

In the breakout room tasked with brainstorming strategies for diverse trainees to cope with bias, ideas focused on actionable steps that trainees can take to build their support network and respond to instances of bias. Specifically, participants detailed the need to develop friends and allies who are willing to speak up or intervene and noted the "chain reaction" that can occur among others when one person in a group is willing to model that they are an ally. Participants also brainstormed specific strategies for responding to inappropriate comments at the moment; for example, by asking clarifying question in response, such as "what do you mean by that?" - which may seem less aggressive for a trainee who is not comfortable being straightforward.

The discussion for faculty mentors to help diverse trainees cope with bias focused largely on the role that mentors can play as advocates at the institutional level (i.e., pushing for systemic change to address the harmful impacts of bias on diverse trainee development and acting as personal advocates in a sponsorship role).
To assist faculty with doing so, participants in this group identified the importance of both empowering faculty with the skills and know-how to advocate for students, as well as protecting faculty from internal pushback from administrators or punitive reactions from supervisors who are not supportive of these changes. In addition, participants recommended that faculty mentors of diverse trainees be given tools to bridge the gap from simple awareness of their own internal biases to how to actually support their trainees in practice. Finally, participants recommended more formal acknowledgment and even payment for faculty and trainee diversity efforts (i.e., serving on diversity committees, helping with minority recruitment and retention efforts).

\section{Assertive communication}

In brainstorming ideas for trainees to develop assertive communication, the discussion focused on strategies for both listening and speaking. For speakers, the group suggested using the "sandwich technique" of starting feedback with a light tone, then presenting the core critique, and then ending on a similarly light note. The group's ideas for trainees when receiving feedback or communication perceived as aggressive included reminding oneself that communication is not personal, asking oneself whether that is how the presenter talks in other contexts or to other people and to consider if content would be interpreted in the same way if delivered by different person (e.g., a male colleague instead of a female colleague).

For mentors helping trainees to communicate assertively instead of aggressively, group participants discussed the role of mentors in normalizing the response of "I don't know" and reminding trainees that developing assertiveness starts from within. In addition, mentors should stress that communication styles differ across groups of people and that a certain degree of code switching is to be expected depending on a person's rank, stature, and discipline. Group participants also discussed practical techniques for trainees to communicate assertively, including repeating oneself at the beginning and end of a statement to get their main point across. Validated communication tools and frameworks such as TeamSTEPPS ${ }^{\circledR}$, in which groups explicitly discuss ground rules and customs for team communication, were also seen as helpful.

\section{Knowing how things work}

Top ideas for trainees from participants in the "knowing how things work," or understanding idiosyncratic institutional norms and the nuts and bolts of research, included supporting students from cultural backgrounds outside the United States to understand common social norms and to improve the lack of transparency on the process of submitting grants or applying to jobs. Other discussion topics included issues that may arise with research support staff, who some participants perceived as being reluctant to invest in getting to know trainees as they rotate in and out of labs or research groups, as well as fostering a normative culture where trainees are not afraid to admit to what they don't know and ask for help or training when needed.

With respect to advice for mentors, participants suggested giving trainees the opportunities to practice and refine skills with supportive feedback, teaching trainees to embrace mistakes and not being afraid of asking "dumb" questions and to encourage institutional leaders to implement training for mentors so they can better support students in developing knowledge and skills to navigate the institution or research environment. Participants also suggested that more senior graduate students can play a role in helping new trainees learn the ropes of their new program. Finally, 
(A)

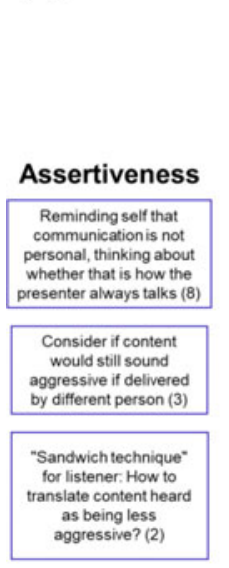

Top Ideas for Trainees

Cope with bias

\begin{tabular}{|c|c|c|}
\hline $\begin{array}{c}\text { Ask } \\
\text { clarifying } \\
\text { questions in } \\
\text { response to } \\
\text { bias (9) }\end{array}$ & $\begin{array}{l}\text { Develop } \\
\text { allies - allies } \\
\text { need to be } \\
\text { willing to } \\
\text { speak up (8) }\end{array}$ & $\begin{array}{l}\text { Find friends } \\
\text { to share } \\
\text { experiences } \\
\text { with and } \\
\text { create a safe } \\
\text { space (6) }\end{array}$ \\
\hline
\end{tabular}

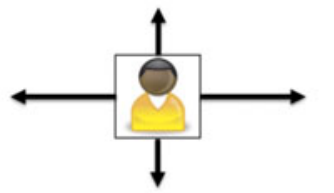

Knowing how things work

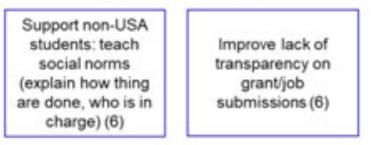

(B)
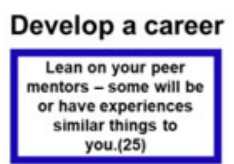

similar things to you.(25)

Find and establish a
mentoring team that meets your needs (17)

Knowing people
Outside your institution Outside your institution.
Networkll (16)

Do what you like, because $e$ if you like it, you'll be good at it and you'll be gain respect for it. (7)



(C)

Top Ideas for Institutions
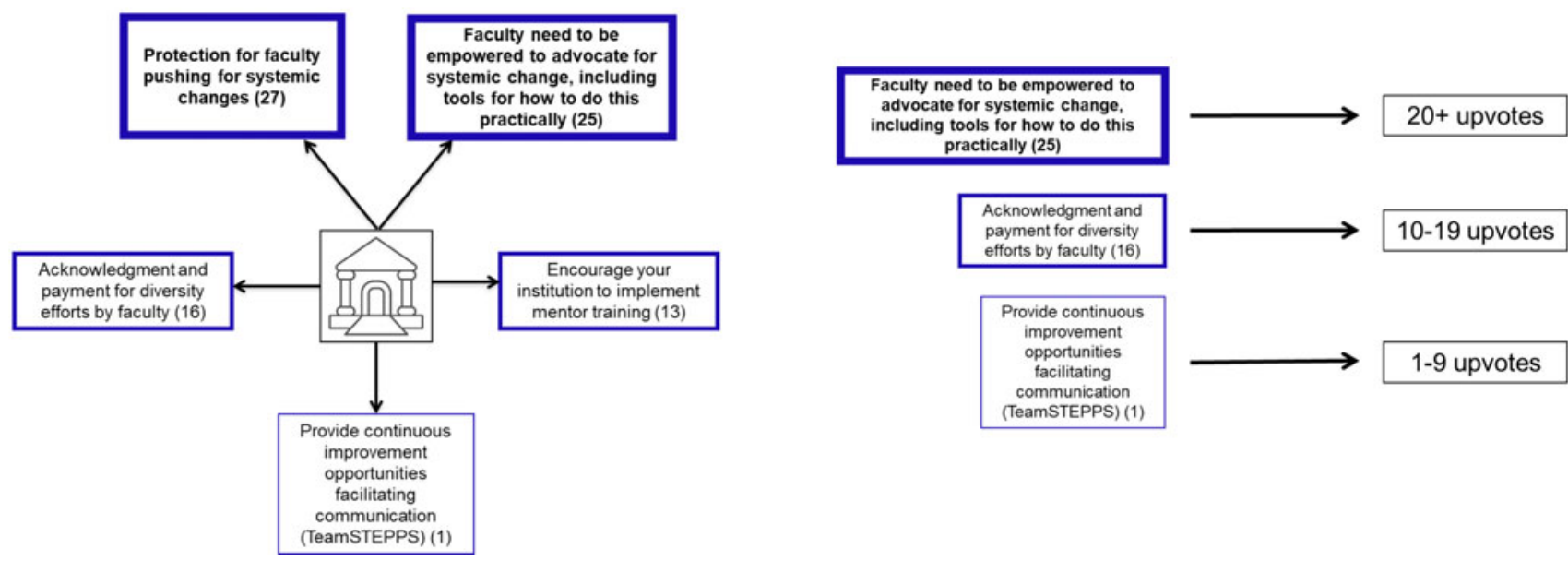

Fig. 2. Top ideas generated for trainees, mentors, and institutions. (A) Top ideas for trainees identified in session breakout rooms. (B) Top ideas for mentors identified in session breakout rooms. (C) Top ideas for institutions identified in session breakout rooms. Note: Numbers in parentheses indicate number of upvotes for a given statement.

participants noted that faculty mentors should be aware that trainees will observe how they handle themselves and therefore should be intentional as a role model in their interactions and behaviors.

\section{Developing a career}

Participants identified specific advice for trainees as they develop a research career, including the importance of finding and establishing a mentoring team that meets the trainee's needs, leaning on peer mentors, who may have had similar experiences; developing relationships with mentors and peers outside of one's home institution; and, finally, to pursue and cultivate research topics and activities that the trainee enjoys, because these will more naturally lend themselves to long-term success.

For mentors to support trainees, participants recommended that faculty promote vulnerability through demystifying the ups and downs of their own career trajectory. Participants also recommended allowing trainees make their own mistakes but to support them with gentle feedback. Participants also suggested that mentors develop clear ideas around sponsorship, specifically by indicating that mentees should try to bring some value to collaboration and that they should have a clear "ask" for sponsorship. Finally, participants discussed how faculty should help mentees develop a broader mentoring team by connecting them to other potential mentors, emphasizing that the mentor can be a point person but mentoring is best in a team, and for the mentor to be honest about their limitations, whether these are limitations on the mentor's time, knowledge, or both.

\section{Wildcard group}

The "wildcard" breakout group was tasked with brainstorming hidden curriculum strategies for diverse trainees and their mentors not highlighted in other breakout groups. Participants in the wildcard group were asked to generate ideas for other hidden curriculum topics featured in the presentation (see Table 1 for full list). Top ideas from this group focused on trainee thoughts and actions, including observing the environment, sit back, and take it in before going all in; the critical role of finding a peer ally or near-peer mentor; accept that trainees may need to forget prior learning that are inhibiting their current success; and that trainees can and should adapt behavior based on the circumstances, but should not compromise who they are fundamentally. 


\section{Discussion}

In this paper, we describe findings from a novel presentation and workshop on diversity-related hidden curriculum. The goal of the study was to produce participant-generated solutions to help doctoral trainees from diverse backgrounds unpack and negotiate these unspoken norms of academia. Results indicate that both mentors and trainees are highly aware of the role that the hidden curriculum plays in shaping student experience and success, even if respondents previously were not familiar with the language used to describe this phenomenon. In addition, mentors and trainees alike voiced the need for institutions to support changes that will facilitate mentors' capacity to address the hidden curriculum with their trainees as well as student success in navigating the implicit norms of academia. This latter finding is particularly noteworthy, as the workshop was specifically structured to brainstorm ideas to help mentors and mentees at the individual and interpersonal levels. However, participants naturally gravitated toward discussions of institutional responsibility to facilitate these changes.

Notably, the hidden curriculum is the most hidden for students from diverse backgrounds not widely represented in academia [17]. Therefore, to improve the experiences and outcomes of doctoral training for students from diverse backgrounds, it is necessary to develop interventions targeting institutions, mentors, and trainees themselves that are focused on demystifying the research hidden curriculum. Strong and culturally responsive mentorship has previously been linked to doctoral student success and persistence in academia for trainees from underrepresented backgrounds [19-21]. Many of the ideas brainstormed by participants in our workshop were actionable suggestions for individual faculty mentors to improve their ability to help students navigate the hidden curriculum of research. However, many faculty mentors of diverse trainees may be unaware of the extent to which personal identity can affect a trainee's experience in academia, particularly if mentors are not themselves from underrepresented backgrounds [22,23]. First, addressing the influence of race, gender, cultural heritage, and other personal identities in doctoral student success may be a necessary step for many research mentors and can be achieved through activities like implicit bias training. More broadly, all ideas to improve faculty awareness of the diversity-related hidden curriculum also serve as ally development activities for faculty members who are not themselves from diverse backgrounds.

Uncovering and navigating these hidden curricula has been identified as a critical component of success at all levels of higher education and, in particular, can influence the academic success of students from backgrounds not widely represented in academia [8]. For trainees who have learned negative thoughts or behaviors over time, it is important to identify and overcome these with targeted action-based tools. Anecdotally, faculty note that some trainees received hidden curriculum teaching them to act confident and self-promote no matter what, while others have received implicit messages to always be deferent or passive. In order to develop a career, trainees must know the options for career opportunities and what each option requires, as well as successfully network within their profession to identify specific positions. Individuals who are the first in their family or social network to enter a $\mathrm{PhD}$ program may experience a profound "network gap" in that they are less aware of ways in which professional positions are identified and pursued [24]. In ideal circumstances, faculty mentors can play a key role in helping doctoral students identify and navigate these unwritten norms and expectations.
Many suggestions for faculty mentors emphasized the role of the department or institution in empowering mentors to help diverse trainees. Two suggestions that were widely upvoted during the workshop referenced the need to educate faculty on how they can push for change at their institutions as well as the importance of protections for faculty pushing for systemic changes, who might face resistance or retaliation from administrators wishing to maintain the status quo. Other suggestions focused on institutional changes that would reward or compensate faculty who take on a disproportionate share of mentoring, student recruitment, and committee service. Some responses alluded to the specific impact of the "minority tax," in which faculty (and trainees, to some extent) from diverse backgrounds are expected to participate in diversityfocused committees and initiatives at a considerable cost to time available for research and other activities [25,26]. Institutions have a variety of strategies available to mitigate this "tax," which include building diversity work into promotion and tenure metrics and engaging nonminority faculty in diversity efforts [27].

As shown in our results, institutional action can be an especially strong mechanism to set up sustainable and meaningful change relating to diversity, equity, and inclusion. Concurrent with this work, Mayo Clinic has been working to develop institutional sustained infrastructure toward a more equitable workplace. This is in part due to Mayo's institutional investment in eradicating racism. Within research, there is a goal of requiring a written "contribution toward equity, inclusion, and diversity" statement within appointment and promotion packages. With these statements, staff will demonstrate alignment with documented promotion metrics aligned with this topic. This shift also means that effort aligned with diversity, equity, and inclusion is reviewed by an institutional panel on a regular basis during the promotion process. It also facilitates the dissemination of accomplishments, knowledge, and ideas across the organization.

Clearly, every academic institution has idiosyncratic norms and culture. Further, every new academic has to understand the unique eccentricities of their institutions to thrive. The systemic hidden curricula faced by nonmajority faculty, however, represent a clear and omnipresent danger of simple survival in academia. As demonstrated by our results, there are methods and solutions to demystify and deal with the hidden curricula. Recognition and implementation of these findings in actionable ways are the next steps. We plan to continue along this path by formally teaching hidden curriculum topics to trainees, opening discussions with mentors, and continuing to present opportunities for institutional change to leadership.

\section{Limitations}

This study has important limitations. First, participants were selfselected into the conference workshop and, from there, into individual breakout rooms during the session. Therefore, findings are likely biased toward the viewpoints of faculty and trainees who are interested in and endorse the importance of negotiating diversityrelated hidden curriculum in academia. However, the ultimate goal of the session was to elicit actionable ideas from mentors and trainees on navigating the hidden curriculum. Participants with a prior interest in improving trainee experiences and, perhaps, personal experience with the hidden curriculum are arguably best suited to lead these discussions and generate ideas from which other mentors, trainees, and institutions can learn. In addition, there were limitations to the method of data collection, since participants could leave and reenter the session or choose not to respond to the anonymous Poll Everywhere questions. 
Acknowledgements. The authors would like to thank Adriana Morales Gomez, Linsey Jackson, Aunay Miller, Harvey Huang, Karen DSouza, and Bradley Bowles for their invaluable assistance as moderators during the ACTS conference workshop described in this paper. This publication was supported by CTSA Grant Number TL1 1R002380 from the National Center for Advancing Translational Science (NCATS). Its contents are solely the responsibility of the authors and do not necessarily represent the official views of the NIH.

Disclosures. The authors have no conflicts of interest to declare.

\section{References}

1. Hafferty FW, O'Donnell JF. The Hidden Curriculum in Health Professional Education. Hanover, NH: Dartmouth College Press, 2015.

2. Margolis E. The Hidden Curriculum in Higher Education. New York: Routledge, 2001.

3. Lauer M. Further Demographic Analyses of NIH R01 Grant Outcomes of T32 Postdoctoral Participants. NIH Extramural NEXUS, 2021. (https:// nexus.od.nih.gov/all/2021/07/27/further-demographic-analyses-of-nih-r01grant-outcomes-of-t32-postdoctoral-participants/)

4. Vettese T. Sexism in the academy: women's narrowing path to tenure. $n+1$ [Internet], Spring 2019 [cited Oct 25, 2021]. (https://www.nplusonemag. com/issue-34/essays/sexism-in-the-academy/)

5. Shim RS. Dismantling structural racism in academic medicine: a skeptical optimism. Academic Medicine 2020; 95(12): 1793-1795.

6. Brown N, Leigh J. Ableism in academia: where are the disabled and ill academics? Disability \& Society 2018; 33(6): 985-989.

7. Bickel J. Women in academic medicine. Journal of the American Medical Women's Association 2000; 55(1): 10-12, 19.

8. Smith B. Mentoring at Risk Students Through the Hidden Curriculum of Higher Education. Lanham, MD: Lexington Books, 2013.

9. Lawrence C, Mhlaba T, Stewart KA, Moletsane R, Gaede B, Moshabela M. The hidden curricula of medical education: a scoping review. Academic Medicine 2018; 93(4): 648-656.

10. Lewis T. Professional development of statisticians in the pharmaceutical sector: evolution over the past decade and into the future. Pharmaceutical Statistics: The Journal of Applied Statistics in the Pharmaceutical Industry 2008; 7(3): 158-169.

11. Kenett R, Thyregod P. Aspects of statistical consulting not taught by academia. Statistica Neerlandica 2006; 60(3): 396-411.

12. Pomann G-M, Boulware LE, Cayetano SM, et al. Methods for training collaborative biostatisticians. Journal of Clinical and Translational Science 2021; 5(1): e26. doi: 10.1017/cts.2020.518.

13. Enders FT, Golembiewski EH, Pacheco-Spann LM, Allyse M, Mielke MM, Balls-Berry JE. Building a framework for inclusion in health services research: development of and pre-implementation faculty and staff attitudes toward the Diversity, Equity, and Inclusion (DEI) plan at Mayo Clinic. Journal of Clinical and Translational Science 2021; 5(1): e88, 1-1. doi: $10.1017 /$ cts.2020.575.

14. Calarco JM. The inconsistent curriculum: cultural tool kits and student interpretations of ambiguous expectations. Social Psychology Quarterly 2014; 77(2): 185-209.

15. Williams JC, Dempsey R. What Works for Women at Work: Four Patterns Working Women Need to Know. New York: New York University Press, 2018.

16. Frankel LP. Nice Girls Don't Get the Corner Office: Unconscious Mistakes Women Make that Sabotage their Careers. New York: Grand Central Publishing, 2014.

17. Calarco JM. A Field Guide to Grad School: Uncovering the Hidden Curriculum. Princeton, NJ: Princeton University Press, 2020.

18. Byars-Winston A, Rogers JG. Testing intersectionality of race/ethnicity $\times$ gender in a social-cognitive career theory model with science identity. Journal of Counseling Psychology 2019; 66(1): 30-44.

19. Carlone HB, Johnson A. Understanding the science experiences of successful women of color: science identity as an analytic lens. Journal of Research in Science Teaching 2007; 44(8): 1187-1218.

20. Byars-Winston A, Leverett P, Benbow RJ, Pfund C, Thayer-Hart N, Branchaw J. Race and ethnicity in biology research mentoring relationships. Journal of Diversity in Higher Education 2020; 13(3): 240-253.

21. Byars-Winston A, Womack VY, Butz AR, et al. Pilot study of an intervention to increase cultural awareness in research mentoring: implications for diversifying the scientific workforce. Journal of Clinical and Translational Science 2018; 2(2): 86-94.

22. Davidson MN, Foster-Johnson L. Mentoring in the preparation of graduate researchers of color. Review of Educational Research 2001; 71(4): 549-574.

23. Prunuske AJ, Wilson J, Walls M, Clarke B. Experiences of mentors training underrepresented undergraduates in the research laboratory. $C B E-$ Life Sciences Education 2013; 12(3): 403-409.

24. Morgan A, Clauset A, Larremore D, LaBerge N, Galesic $M$. Socioeconomic Roots of Academic Faculty. SocArXiv. Preprint posted online March 24, 2021. doi: 10.31235/osf.io/6wjxc.

25. Cleveland R, Sailes J, Gilliam E, Watts J. A theoretical focus on cultural taxation: who pays for it in higher education. Advances in Social Sciences Research Journal 2018; 5(10): 95-98. doi: 10.14738/assrj. 510.5293.

26. Akin Y. The time tax put on scientists of colour. Nature 2020; 583(7816): 479-481.

27. Rodríguez JE, Campbell KM, Pololi LH. Addressing disparities in academic medicine: what of the minority tax? BMC Medical Education 2015; 15(1): $1-5$. 